\title{
Development of cell penetrating peptides for effective delivery of recombinant factors into target cells
}

\begin{abstract}
The cell membrane is a protective layer that strictly controls the passage of molecules restricting the delivery of biomolecules such as drugs, oligonucleotides, peptides, and siRNA into the cells. This shortcoming has been overcome by the discovery of Cell-Penetrating Peptides (CPPs) that has undergone 30 years of evolution. To date, CPPs are largely modified to improve its efficacy and to suit the different delivery applications. The modes of CPPs penetration are still an unresolved mystery and requires further investigations to increase its effectiveness and to diversify its use. Despite having huge potential as a biomolecule carrier, CPPs also have some drawbacks. In this review, the natural and synthetic CPPs, the modifications that have been conducted on CPPs to improve its efficacy, its extended applications, modes of penetration and limitation as well as challenges will be discussed.
\end{abstract}

Keyword: Cell-penetrating peptide (CPP); Cell membrane; Biomolecule carrier; Protein transduction technology; Translocation; Recombinant factors 